\title{
Ocular Complications in Assault-Related Blowout Fracture
}

\author{
Woong Kyu Choi, \\ Young Joon Kim, \\ Sang Hyun Nam, \\ Young Woong Choi \\ Department of Plastic and Reconstructive \\ Surgery, Sanggye Paik Hospital, Inje \\ University College of Medicine, Seoul, Korea
}

No potential conflict of interest relevant to this article was reported
Background: Blowout fracture is one of the most common facial fractures, and patients usually present with accompanying ocular complications. Many studies have looked into the frequency of persistent ocular symptoms, but there is no study on assault patients and related ocular symptoms. We evaluated the incidence of residual ocular symptoms in blowout fractures between assaulted and non-assaulted patients, and sought to identify any connection among the degree of enophthalmos, defect size, and assault-related injury. Methods: A retrospective review was performed for any patient who sustained a unilateral blowout fracture between January 2010 to December 2014. The collected data included information such as age, gender, etiology, and clinical ocular symptoms as examined by an ophthalmologist. This data was analyzed between patients who were injured through physical altercation and patients who were injured through other means. Results: The review identified a total of 182 patients. Out of these, 74 patients $(40.7 \%)$ have been struck by a fist, whereas 108 patients (59.3\%) have sustained non-assault related injuries. The average age was 36.1 years, and there was a male predominance in both groups (70 patients [94.6\%] in the assaulted group and 87 patients [80.6\%] in the non-assault group). Diplopia and enophthalmos were more frequent in patients with assault history than in non-assaulted patients $(p<0.05)$. Preoperatively, 25 patients $(33.8 \%)$ with assault history showed diplopia, whereas 20 patients $(18.5 \%)$ showed diplopia in the non-assaulted group $(p<0.05)$. Preoperative enophthalmos was present in 34 patients $(45.9 \%)$ with assault history, whereas 31 patients $(28.7 \%)$ showed enophthalmos in the non-assaulted group $(p<0.05)$.

Conclusion: : Patients with an assault history due to a fist blow experienced preoperative symptoms more frequently than did patients with non-assault-related trauma history. Preoperative diplopia and enophthalmos occurred at a higher rate for patients who were assaulted. Surgeons should take into account such characteristics in the management of assaulted patients.

Keywords: Orbital fractures / Diplopia / Enophthalmos / Violence

\section{INTRODUCTION}

A blowout fracture only involves the walls of the orbit, and occurs in the most fragile part of the orbital wall. Blowout fractures can be associated with intracranial, optic nerve, lacrimal system, eyelid, and globe injuries [1,2]. Patients usually sustain facial fractures

\section{Correspondence: Young Joon Kim}

Department of Plastic and Reconstructive Surgery, Sanggye Paik Hospital, Inje University College of Medicine, 1342 Dongil-ro, Nowon-gu, Seoul 01757, Korea E-mail: s2639@paik.ac.kr

Received March 16, 2016 / Revised May 23, 2016 / Accepted May 23, 2016 from a blunt impact to the orbit and are diagnosed with accompanying ocular symptoms. Such ocular symptoms are inspected and examined by careful physical examinations and computed tomography (CT) scans. Surgical intervention is recommended usually within 2 weeks, to replace the fractured bone segments and correct soft tissue distortion. Even after surgical repair, patients may suffer from permanent complications such as persistent diplopia, visual loss, dystopia, enophthalmos, scarring, and sensory disturbances [2].

Many studies have looked into the frequency of persistent ocu- 
lar symptoms. The majority of patients admitted to the emergency department are those who have sustained an assault, but there is no study on assault patients and related ocular symptoms. In practice, it is difficult to explain to the patients the outcomes and expectation of surgery. Therefore, we evaluated the incidence of residual ocular symptoms in blowout fractures between assaulted and nonassaulted patients, and sought to identify any connection among the degree of enophthalmos, defect size, and assault-related injury.

\section{METHODS}

A retrospective review was performed for any patient who sustained a unilateral blowout fracture between January 2010 to December 2014. Patients were included in the study for having received operative intervention with at least 6 months of follow up. The collected data included information such as age, gender, etiology, and clinical ocular symptoms as examined by an ophthalmologist. Statistical relationship were analyzed among ocular symptoms and injury location, fracture dimension, degree of enophthalmos, etiology, and days to surgery. Ocular symptoms were categorized again into preoperative (acute) symptoms and postoperative (late) complications. Complications were defined as secondary ocular symptoms, which persisted or newly appeared within the 6 months of follow up. All of the patients had been evaluated by both a plastic surgeon and an ophthalmologist in the emergency department. Diagnoses were made using facial 3D CT and clinical examinations. Only pure blowout fracture patients were included, and old fractures or zygomaticomaxillary fractures were excluded. As per the inclusion criteria, all of the patients in the study had undergone corrective surgery under general anesthesia. The blowout fracture was fixed via subciliary, transconjunctival, or transcaruncular approach. Absorbable mesh plates were used to reconstruct the orbital floor and wall, and any herniation of periocular tissue was repositioned prior to placement of the absorbable plate on the orbital floor. All of the patients were followed up for at least 6 months at both the plastic surgery and ophthalmology outpatient clinics. Follow-up visits were made at 2 weeks, 1 month, 3 months, 6 months, and 1 year. Any symptoms either complained by the patient or assessed by a physician were recorded on the medical chart and studied retrospectively.

Patients were divided into subgroup 1 and 2, according the etiology of the injury, assault or non-assault. The definition of ocular signs and symptoms were based on previous publications [3]. The CT examinations had been performed for all patients using the imaging parameters (Toshiba Aquilion One, Tokyo, Japan): 120 kV, 150 mAs, FOV 25 cm, and 3-mm section thickness. Diplopia was measured using the Lees screen test, where the patient fixes one eye at 9 points in an inner square and 17 points in an outer square, and diplopia is measured at a screen placed at a right angle [4]. The amount of motility restriction was measured in vertical, horizontal, and diagonal directions. CT images were used to assess the degree of enophthalmos. To calculate the degree of enophthalmos, we measured the distance from the corneal center to the shortest segment from the lateral orbital rim to the medial orbital margin (Fig. 1). An eye with a difference of more than $2 \mathrm{~mm}$ compared to the normal eye on the axial CT image were considered to have enophthalmos.

In this study, we also measured the defect size of the blowout fracture, by measuring the bony defect in the coronal view of the CT scan (Fig. 2). The thickness of the CT sections was $3 \mathrm{~mm}$, and the bony defect length was set as $\mathrm{C}$. Then, we used the sum of the product between $\mathrm{C}$ and $3 \mathrm{~mm}$ for each slice, $\sum_{n=1}^{N} C_{n} * 3$, to obtain the defect size.

The independent $\chi^{2}$-test and Fisher's exact test were used to compare the difference between the two groups. Linear regression analysis was used to find the relationship between bony defect size and degree of enophthalmos. Statistical analysis was performed using PASW ver. 18.0 (SPSS Inc., Chicago, IL, USA).

\section{RESULTS}

\section{Patient demographics}

The review identified 182 patients who met the inclusion criteria. Out of these, 74 patients (40.7\%) have been struck by a fist blow, whereas 108 patients (59.3\%) have sustained non-assault related injuries. The average age was 36.1 years, and there was a male predominance in both groups (70 patients [94.6\%] in the assaulted 


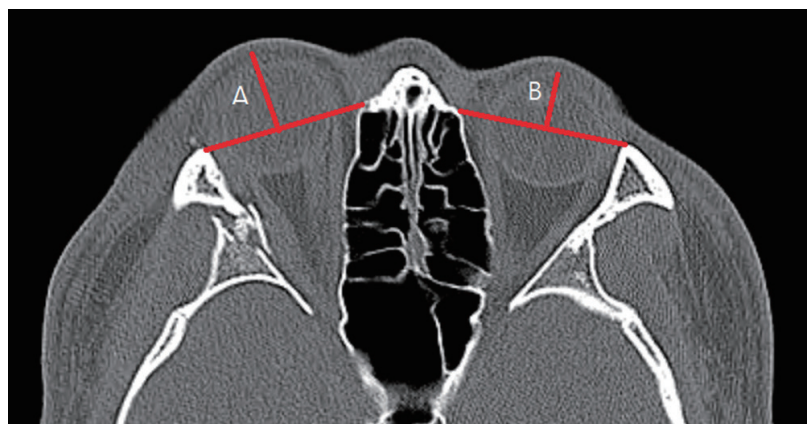

Fig. 1. Measurement of the degree of enophthalmos. A, normal eye; B, injured eye.

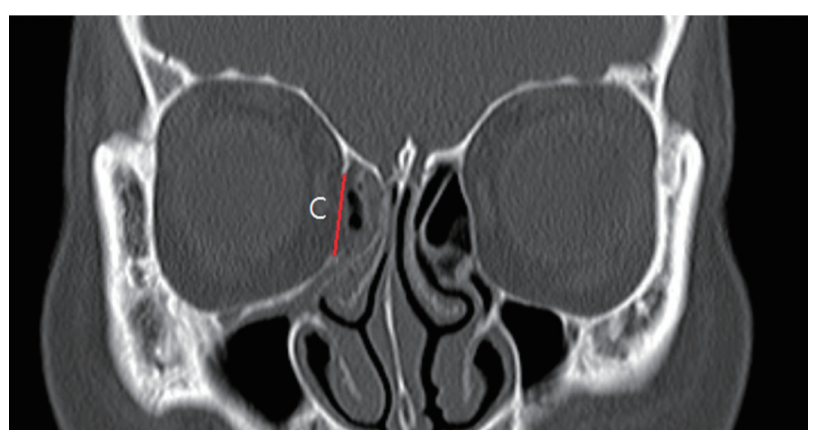

Fig. 2. Measurement of the bony defect. C, length of the medial wall defect.

group and 87 patients [80.6\%] in the non-assault group). The average age was 30.54 in the assault group, and 36.31 in the non-assault group (Table 1). All of the patients included in this study were diagnosed with isolated blowout fracture. Of the whole, $99 \mathrm{pa}-$ tients sustained injury on the left side whereas 83 patients were injured on the right orbit. Medial blowout fractures were present in 109 patients; 40 patients had orbital floor fractures; and, 33 patients had combined inferomedial orbital fractures. The injuryto-operation was 6.67 days.

\section{Preoperative (acute) symptoms}

Diplopia and enophthalmos were more frequent in patients with assault history than in non-assaulted patients $(p<0.05)$. Preoperatively, 25 patients (33.8\%) with assault history showed diplopia, whereas 20 patients (18.5\%) showed diplopia in the non-assaulted group $(p<0.05)$. Preoperative enophthalmos was present in 34 patients (45.9\%) with assault history, whereas 31 patients (28.7\%) showed enophthalmos in the non-assaulted group $(p<0.05)$ (Table 2). Other
Table 1. Demographics of the 182 patients included in the study

\begin{tabular}{lccc}
\multirow{2}{*}{ Variables } & \multicolumn{2}{c}{ Assault history } & \\
\cline { 2 - 3 } Sex & Yes $(n=74)$ & No $(n=108)$ & \\
$\quad$ Male & & & 0.008 \\
$\quad$ Female & $40(94.6)$ & $87(80.6)$ & \\
Age (yr) & $4(5.4)$ & $21(19.4)$ & \\
Direction & $30.54 \pm 13.85$ & $37.39 \pm 18.00$ & 0.004 \\
Left & & & 0.880 \\
Right & $41(55.4)$ & $58(53.7)$ & \\
Location & $33(44.6)$ & $50(46.3)$ & \\
Inferior & & & 0.116 \\
$\quad$ Medial & $18(24.3)$ & $22(20.4)$ & \\
Inferior+medial & $38(51.4)$ & $71(65.7)$ & \\
\hline
\end{tabular}

A $p$-value was calculated by independent $\chi^{2}$-test, Fisher's exact test. ${ }^{a} p$-value was calculated by t-test.

Table 2. Preoperative symptoms

\begin{tabular}{|c|c|c|c|}
\hline \multirow{2}{*}{ Variables } & \multicolumn{2}{|c|}{ Assault history } & \multirow{2}{*}{$p$-value } \\
\hline & Yes $(n=74)$ & No $(n=108)$ & \\
\hline $\begin{array}{l}\text { Subconjunctival } \\
\text { hemorrhage }\end{array}$ & & & 0.071 \\
\hline Yes & 22 (29.7) & $19(17.6)$ & \\
\hline No & 52 (70.3) & 89 (82.4) & \\
\hline Diplopia & & & 0.023 \\
\hline Yes & 25 (33.8) & 20 (18.5) & \\
\hline No & 49 (66.2) & 88 (81.5) & \\
\hline Enophthalmos & & & 0.019 \\
\hline Yes & $34(45.9)$ & $31(28.7)$ & \\
\hline No & $40(54.1)$ & 77 (71.3) & \\
\hline $\begin{array}{l}\text { Extraocular movement } \\
\text { limitation }\end{array}$ & & & 0.170 \\
\hline Yes & $24(32.4)$ & 24 (22.2) & \\
\hline No & $50(67.6)$ & 84 (77.8) & \\
\hline Blurred vision & & & 0.551 \\
\hline Yes & $6(8.1)$ & $6(5.6)$ & \\
\hline No & 68 (91.9) & $102(94.4)$ & \\
\hline $\begin{array}{l}\text { Infraorbital nerve } \\
\text { hypoesthesia }\end{array}$ & & & 0.492 \\
\hline Yes & $7(9.5)$ & $14(13.0)$ & \\
\hline No & $67(90.5)$ & $94(87.0)$ & \\
\hline
\end{tabular}

A $p$-value was calculated by independent $\chi^{2}$-test, Fisher's exact test.

ocular findings such as blurred vision and infraorbital nerve hypoesthesia did show a difference between the groups, but this was not statistically significant. 


\section{Postoperative (late) symptoms}

Compared to preoperative symptoms, postoperative complications did not show statistically significant difference between the two groups (Table 3). Postoperative diplopia, which was examined and followed up for at least 6 months after the surgery, was observed similarly in the both groups, 10 patients (13.5\%) in the assaulted group, and 15 patients (13.9\%) in the non-assaulted group. Traumatic optic neuropathy was observed in 11 (14.9\%) of the assaulted patients and in 20 (18.5\%) of the non-assaulted patients. Extraocular movement (EOM) limitation was observed in 15 (20.3\%) of the assaulted group and in 17 (15.7\%) of the non-assaulted patients. Infraorbital nerve hypoesthesia was observed in 7 (9.5\%) of the assaulted patients and in 10 (9.3\%) of the non-assaulted group. Postoperative ectropion was not observed in the assault group, whereas 4 patients experienced ectropion in the non-assault group. None of the differences in postoperative complications was statistically significant (all $p>0.05$ ).

Table 3. Postoperative complications

\begin{tabular}{|c|c|c|c|}
\hline \multirow{2}{*}{ Variables } & \multicolumn{2}{|c|}{ Assault history } & \multirow{2}{*}{$p$-value } \\
\hline & Yes $(n=74)$ & No $(n=108)$ & \\
\hline $\begin{array}{l}\text { Traumatic optic } \\
\text { neuropathy }\end{array}$ & & & 0.554 \\
\hline Yes & $11(14.9)$ & $20(18.5)$ & \\
\hline No & $63(85.1)$ & 88 (81.5) & \\
\hline Diplopia & & & 0.879 \\
\hline Yes & $10(13.5)$ & 15 (13.9) & \\
\hline No & 64 (86.5) & 93 (86.1) & \\
\hline Enophthalmos & & & 0.219 \\
\hline Yes & $13(17.6)$ & $18(16.7)$ & \\
\hline No & $61(82.4)$ & 90 (83.3) & \\
\hline $\begin{array}{l}\text { Extraocular movement } \\
\text { limitation }\end{array}$ & & & 0.553 \\
\hline Yes & $15(20.3)$ & $17(15.7)$ & \\
\hline No & 59 (79.7) & 91 (84.3) & \\
\hline $\begin{array}{l}\text { Infraorbital nerve } \\
\text { hypoesthesia }\end{array}$ & & & 0.964 \\
\hline Yes & $7(9.5)$ & $10(9.3)$ & \\
\hline No & $67(90.5)$ & $98(90.7)$ & \\
\hline Ectropion & & & 0.147 \\
\hline Yes & 0 & $4(3.7)$ & \\
\hline No & $74(100.0)$ & $104(96.3)$ & \\
\hline
\end{tabular}

A $p$-value was calculated by independent $\chi^{2}$-test, Fisher's exact test.

\section{Defect size and degree of enophthalmos}

The assaulted patient group had an average bone defect size of 49.41 $\mathrm{mm}^{2}$, whereas the non-assaulted group had a mean bone defect size of $39.14 \mathrm{~mm}^{2}$ (Table 4). The degree of enophthalmos was statistically significant between the two groups. There were a total of 34 (45.9\%) patients diagnosed with enophthalmos in the assaulted group, and only 31 (28.7\%) patients in the non-assaulted group $(p<0.05)$. Assaulted patients had a mean $3.28 \mathrm{~mm}$ of enophthalmos, whereas non-assaulted patients had only $2.78 \mathrm{~mm}(p<0.05)$.

Comparing the two groups preoperatively and postoperatively, the degree of enophthalmos was reduced from $3.28 \mathrm{~mm}$ to 2.44 $\mathrm{mm}$ in the assaulted group $(p<0.05)$ and from $2.78 \mathrm{~mm}$ to 2.32 $\mathrm{mm}$ in the non-assaulted group $(p<0.05)$. In the assaulted group, the total number of patients diagnosed with enophthalmos was 34 (45.9\%) preoperatively, which was reduced to 15 (20.3\%) postoperatively $(p<0.05)$. In the non-assaulted group, the total number of patients diagnosed with enophthalmos was 31 (28.7\%) preoperatively, which was reduced to $16(14.8 \%)$ postoperatively $(p<0.05)$ (Table 5).

The correlation between bone defect size and degree of enophthalmos was analyzed only preoperatively, since there is no bone defect postoperatively. There was a significant positive linear correlation between the two variables in both groups (Fig. 3). The degree of enophthalmos was $\mathrm{E}=0.015 \mathrm{~A}+0.538$ ( $\mathrm{E}$, the degree of enophthalmos; A, the area of the bone defect) in the assaulted group, and $\mathrm{E}=0.010 \mathrm{~A}+0.401$ in the non-assaulted group $(p<0.05)$.

Table 4. Comparison of bone defect size and enophthalmos degree according to assault history

\begin{tabular}{|c|c|c|c|}
\hline \multirow{2}{*}{ Variables } & \multicolumn{2}{|c|}{ Assault history } & \multirow{2}{*}{$p$-value } \\
\hline & Yes $(n=74)$ & No $(n=108)$ & \\
\hline $\begin{array}{l}\text { Bone defect size } \\
\left(\mathrm{mm}^{2}\right)^{\mathrm{a})}\end{array}$ & $49.41 \pm 62.45$ & $39.14 \pm 59.73$ & 0.265 \\
\hline $\begin{array}{l}\text { Degree of } \\
\text { enophthalmos } \\
(\mathrm{mm})^{a)}\end{array}$ & $3.28 \pm 1.55$ & $2.78 \pm 1.32$ & 0.024 \\
\hline Enophthalmos & & & 0.019 \\
\hline Yes & 34 (45.9) & 31 (28.7) & \\
\hline No & $40(54.1)$ & 77 (71.3) & \\
\hline
\end{tabular}


Table 5. Comparison of enophthalmos and degree of enophthalmos preoperatively and postoperatively

$\begin{array}{lrrr}\text { Assault history } & \text { Preoperative } & \text { Postoperative } & p \text {-value } \\ \text { Yes }(\mathrm{n}=74) & & & \\ \quad \begin{array}{l}\text { Degree of enophthalmos (mm) } \\ \text { Enophthalmos }\end{array} & 3.28 \pm 1.55 & 2.44 \pm 0.88 & <0.001 \\ \quad & & 0.002 \\ \quad \text { Yes } & 34(45.9) & 15(20.3) & \\ \quad \text { No } & 40(54.1) & 59(79.7) & \\ \text { No ( } \mathrm{n}=108) & & & \\ \text { Degree of enophthalmos (mm) } & 2.78 \pm 1.32 & 2.32 \pm 0.77 & <0.001 \\ \text { Enophthalmos } & & & 0.020 \\ \quad \text { Yes } & 31(28.7) & 16(14.8) & \\ \quad \text { No } & 77(71.3) & 92(85.2) & \\ & & & \\ & & \end{array}$

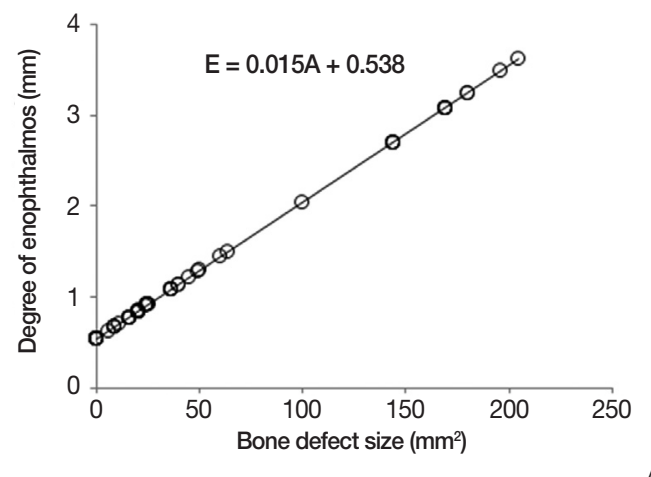

A

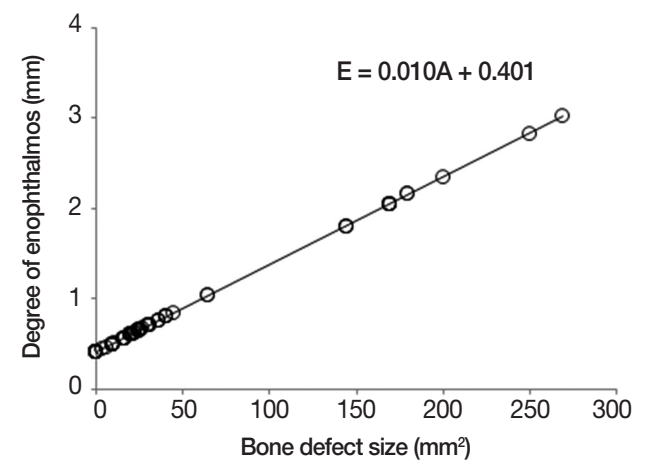

B

Fig. 3. The correlation between bone defect size and degree of enophthalmos. (A) Assaulted patient group. (B) Non-assaulted patient group. E, the degree of enophthalmos; A, the area of the bone defect.

\section{DISCUSSION}

Blowout fractures are one of the most common facial bone fractures and can cause serious ocular complications in patients. Surgeons replace the brittle fractured segments with absorbable biodegradable mesh plates or other implants to minimize complications and restore function to prevent or solve ocular problems, but the reconstruction can also create problems for the patients [5]. Hence, there is no universal guideline regarding the treatment, optimal timing of surgery, or management of surgical outcomes [6]. We felt that it would be meaningful to retrospectively analyze the cause of injury and statistically analyze it to find any correlation. Furthermore, we sought to assess blowout fracture patients presenting with symptoms to find out if there is a meaningful difference between assault patients and patients of a different traumatic etiology.

In previous studies, many authors reported varying degrees of postoperative sequelae. According to Folkestad and Westin [7], postoperative permanent sequelae were found in $83 \%$ of the patients. The most common sequelae were sensibility disturbances (55\%), vision symptoms (48\%), and cosmetic problems (44\%). Permanent diplopia was noted in $17 \%$ of the patients who underwent surgery. Other authors such as Neovius et al. [8] report that persistent diplopia was present in 3.7\% of fractures involving the orbit. In other studies where all fractures involving the orbit were included, permanent diplopia was reported to be $6 \%$ to $15 \%[3,5]$. The mean age of the patients in our study was 36.1, which is comparable to that reported in the literature [3-5,9]. The patient group is relatively young, and permanent diplopia can be a substantial problem for such patients.

Previous studies have reported delayed repair, volume of fracture, extraocular muscle swelling, fracture site, and patient age as potential risk factors for persistent diplopia after surgery $[10,11]$. According to Tahiri et al. [9], however, the most important independent and statistically significant predictor of postoperative diplopia is the presence of preoperative diplopia. In our study, assaulted patients experienced preoperative diplopia more frequently than did non-assaulted patients $(p<0.5)$, but postoperatively, there was no statistically significant difference between the groups. Many authors have suggested this observation to be due to EOM swelling since the injury, and preoperative swelling of the EOM on the CT scan has shown to be a significant risk factor [12]. We agree with Jin and preoperative swelling of the EOM should not be dismissed upon initial CT examination.

In our study, preoperative enophthalmos was significantly different between the two groups. Enophthalmos was more pro- 
nounced in the assaulted group, with 19 patients having preoperative enophthalmos and diplopia simultaneously. According to Converse and Smith [13], the key mechanism of orbital fracture is increased intraorbital hydraulic pressure. In a blunt force trauma due to a fist blow, the force transcends into the orbit and causes a blowout fracture. A fist blow is considered a low-velocity injury [9], and Fujino et al. [14] have previously demonstrated that the hydraulic mechanism requires 10 times as much force to produce a blowout fracture than by the buckling mechanism. Therefore, the low-velocity fist blow is likely to have caused a transcending force throughout the orbit and created enophthalmos. It seems plausible that these two symptoms are associated with one another in fist blow injuries.

According to Putterman et al. [15], adult patients with isolated blowout fractures will eventually recover from ocular motility limitations over time; however, surgical intervention is necessary for severe blowout fractures with soft tissue entrapment on CT scan [16]. All of the patients included in this study had preoperative CT scans prior to surgery, and soft tissue herniation were observed in all patients to varying degrees. In this study, we hypothesized that assault patients who underwent surgery will have different characteristics before or after surgical intervention. Most of our data was consistent with those from previous studies, but we found that assault injuries increase the likelihood of preoperative diplopia and enophthalmos. According to Tahiri et al. [9], preoperative diplopia is a risk factor for persistent diplopia. Surgeons should take into account such characteristics of assault patients and seek a more objective method of evaluating ocular symptoms. Some ocular findings, such as diplopia, is subjective and difficult to quantify [4]. Hess Aria Ratio is an objective method of examining diplopia according to Fujino et al. [14] and should be utilized by specialists to accurately assess diplopia preoperatively and postoperatively.

Upon analyzing the CT images, we found that there is a significant difference of the degree of enophthalmos in assaulted patients (Table 4). Bone defect size did not show significant difference between the two groups, however. The degree and the presence of enophthalmos are greater in assaulted patients compared to non-assaulted patients. We believe that this phenomenon is due to the transcending force from a fist blow. Additionally, our study finds a positive correlation between bone defect and the degree of enophthalmos. We can utilize the formula mentioned earlier to roughly estimate the bone defect area in $\mathrm{mm}^{2}$. The following formula can be used for assaulted patients: $\mathrm{E}=0.015 \mathrm{~A}+0.538$ (E, the degree of enophthalmos; A, the area of the bone defect). On the other hand, the formula $\mathrm{E}=0.010 \mathrm{~A}+0.401$ can be used for the non-assaulted group. Such formulas can be used easily in clinical settings, and our results indicate that there is a stronger positive correlation in assaulted patients.

This retrospective study has some limitations, since patients had been examined by different physicians. Another limitation is that we could not rule out diplopia due to nerve injuries. Symptoms such as diplopia and blurry vision are subjective symptoms, and we had to partly depend on history taking, which might have lowered the reliability of this study. Such symptoms are also likely to be temporary findings, which result as the consequences of soft tissue swelling, contusion, or intramuscular hematoma. Facial CT scans were taken on the day of injury and also after surgery. The main reason for the postoperative CT scan was to rule out any possibility of retrobulbar hematoma, which is a serious complication that can result in blindness. Thus, we were unable to perform follow up CT scans 6 months after the surgery, due to insurance coverage issues, and had to rely on physical examination for longterm data. Despite these limitations, we found that assault patients did show significant difference in the degree of enophthalmos and diplopia, and this phenomenon was associated with relatively larger defect sizes and degree of enophthalmos.

In blowout fracture patients who have sustained a fist blow to the orbit, we suggest facial 3D scans to assess soft tissue herniation and fracture dimensions. Accurate assessment of diplopia and enophthalmos is also necessary in order to predict persistent ocular symptoms and educate the patient of postoperative expectations. Additionally, a thorough understanding of the specific trauma mechanisms and resulting injury patterns is imperative for surgeons. We believe our study will be beneficial to plastic surgeons and many specialists in diagnosing and treating assault related blowout fracture patients. 


\section{REFERENCES}

1. Burm JS, Chung CH, Oh SJ. Pure orbital blowout fracture: new concepts and importance of medial orbital blowout fracture. Plast Reconstr Surg 1999;103:1839-49.

2. Dortzbach RK, Kikkava DO. Blowout fractures of the orbitalfloor. In: Stewart WB, editor. Surgery of the eyelid, orbit and lacrimal system: ophthalmology monograph 8. San Francisco: American Academy of Ophthalmology; 1995. p. 204-23.

3. al-Qurainy IA, Stassen LF, Dutton GN, Moos KF, el-Attar A. The characteristics of midfacial fractures and the association with ocular injury: a prospective study. Br J Oral Maxillofac Surg 1991;29:291-301.

4. Amrith S, Almousa R, Wong WL, Sundar G. Blowout fractures: surgical outcome in relation to age, time of intervention, and other preoperative risk factors. Craniomaxillofac Trauma Reconstr 2010;3:131-6.

5. al-Qurainy IA, Stassen LF, Dutton GN, Moos KF, el-Attar A. Diplopia following midfacial fractures. Br J Oral Maxillofac Surg 1991;29:302-7.

6. Vallier HA, Wang X, Moore TA, Wilber JH, Como JJ. Timing of orthopaedic surgery in multiple trauma patients: development of a protocol for early appropriate care. J Orthop Trauma 2013;27:543-51.

7. Folkestad L, Westin T. Long-term sequelae after surgery for orbital floor fractures. Otolaryngol Head Neck Surg 1999;120:914-21.

8. Neovius E, Fransson M, Matthis SP, Persson C, Ostlund S, Farnebo F, et al. Persistent diplopia after fractures involving the orbit related to nerve injury. J Plast Reconstr Aesthet Surg 2015;68:219-25.

9. Tahiri Y, Lee J, Tahiri M, Sinno H, Williams BH, Lessard L, et al. Preoperative diplopia: the most important prognostic factor for diplopia after surgical repair of pure orbital blowout fracture. J Craniofac Surg 2010;21:1038-41.

10. Hosal BM, Beatty RL. Diplopia and enophthalmos after surgical repair of blowout fracture. Orbit 2002;21:27-33.

11. Biesman BS, Hornblass A, Lisman R, Kazlas M. Diplopia after surgical repair of orbital floor fractures. Ophthal Plast Reconstr Surg 1996;12:9-16

12. Jin HR, Lee HS, Yeon JY, Suh MW. Residual diplopia after repair of pure orbital blowout fracture: the importance of extraocular muscle injury. Am J Rhinol 2007;21:276-80.

13. Converse JM, Smith B. Enophthalmos and diplopia in fractures of the orbital floor. Br J Plast Surg 1957;9:265-74.

14. Fujino T. Experimental "blowout" fracture of the orbit. Plast Reconstr Surg 1974;54:81-2.

15. Putterman AM, Stevens T, Urist MJ. Nonsurgical management of blow-out fractures of the orbital floor. Am J Ophthalmol 1974;77:232-9.

16. Gilbard SM, Mafee MF, Lagouros PA, Langer BG. Orbital blowout fractures: the prognostic significance of computed tomography. Ophthalmology 1985;92:1523-8. 